\title{
The Manchester regional screening programme: a 10-year exercise in patient and family care
}

\section{Summary and conclusions}

Up to the end of 1978 the Willink Biochemical Genetics Unit had screened 506821 babies for metabolic abnormalities over 10 years-98-99\% of the children born in the region. Sixty-nine cases of phenylketonuria (PKU), 42 cases of histidinaemia, and six cases of homocystinuria . were detected. As well as treating affected children, the staff of the unit have concentrated on providing full support for their families and maintaining good communications with parents, general practitioners, health visitors, and midwives. A clinic liaison sister has provided valuable support for health visitors and an important link between the unit and community services. A study of the costs of screening and treating cases of PKU for the year 1978 showed that this was cheaper, by $£ 569000$, than the costs of looking after patients with untreated PKU.

\section{Introduction}

There is a tendency to think of screening programmes for metabolic disorders as laboratory- or computer-based exercises. But the 10 years' experience of the Manchester unit has clearly shown the need for such a unit to be clinically oriented, with adequate facilities for treating the patient and for providing support for the whole family in their day-to-day life.

The early work of the Willink Biochemical Genetics Unit has been described, ${ }^{1}$ - together with the underlying philosophy in screening for inherited disease in the community. After 10 years and the first half million babies tested it is possible to evaluate the work that has been done.

\section{Work of the unit}

The initial criteria have been met. The unit has screened around 98-99" " of the children born in the region; the method used, onedimensional paper chromatography of heparinised blood ${ }^{3}$ taken between days 10 and 14, has been easy to handle, reliable, and cheap. So far as is known there have been no false-negative results, and the most recent costing, even in these inflationary days, is $37 \mathrm{p}$ per baby tested. There were a few false-positive results. There have been certain difficulties with collecting and transporting blood and its management in the laboratory (inadequate specimens, leakage of blood from tubes, breakages). This, plus the need to retest the doubtful cases, has resulted in a repeat rate of $3.5^{\circ},{ }^{\circ}$ to $4^{\prime \prime}$, during the last few years.

Up to 31 December 1978, 506821 babies were fully tested: 69 cases of phenylketonuria (PKU) were detected (an incidence of 1 in

\section{Royal Manchester Children's Hospital, Manchester M27 1HA}

G M KOMROWER, MA, FRCP, honorary consultant paediatrician, former director Willink Biochemical Genetics Unit

I B SARDHARWALLA, FRCP, DCH, consultant paediatrician, director Willink Biochemical Genetics Unit

B FOWLER, MIBIOL, PHD, senior biochemist, Willink Biochemical Genetics Unit

C BRIDGE, LIBIOL, senior medical laboratory scientific officer, Willink Biochemical Genetics Unit
7345 ), as well as 42 cases of histidinaemia ( 1 in 12067) and six cases of homocystinuria ( 1 in 84470 ). Thus the main volume of work has been with phenylketonuric children and to a lesser extent with patients with histidinaemia, although the work with children with homocystinuria has, in a qualitative sense, been much more demanding.

\section{Pattern of working}

In addition to the administrative and technical arrangements for adequate screening, the unit planned to confirm diagnoses promptly in cases under suspicion and to offer immediate treatment to affected children. This included appropriate dietetic and nutritional advice and an explanation of the disorder of the family. We quickly realised that the treatment offered, although immediately satisfactory for the patient, was not enough, as the whole family needed considerable and constant psychological support and encouragement. To this end good communication was ensured between the unit and the family, the general practitioner, and the health visitor.

To achieve this the management of such cases was concentrated into one regional unit. The entire unit was put into one building, where the metabolic ward, the outpatient consulting room, the laboratory, the dietitian's room, and the administrative offices were all adjacent. This has promoted a good atmosphere among the various members of the unit, who inevitably are in regular daily contact, and has helped the families who at each visit go through the same door for all their consultations and tests and know the laboratory and office staffs by sight if not always by name.

All parents are asked to sign their consent before the screening test is done. Reports of all tests are sent out. The negative results are sent each week to the administrative district or area concerned, who tell the parents of the results through their health visitors. Despite this, 50 calls are received each week from health visitors who have not yet had a result from their central office and who are anxious to know it before they visit the family again. When the result is doubtful or the specimen is unsatisfactory the health visitor concerned is telephoned and she arranges to collect a second specimen. The taking of blood upsets many parents, especially when the test has to be repeated, so our general policy allows the health visitor to repeat the test once but requires the parents to bring the child to the unit if further study is needed. At this visit the family is seen by a consultant, who gives a full explanation, examines the baby carefully, and arranges for a detailed study of the plasma and urine. This somewhat time-consuming arrangement has been greatly appreciated by the parents.

The results of the positive tests are given by telephone to the general practitioner and health visitor, who tell the family immediately and arrange for the infant to be brought to the hospital, where they are met by the liaison nursing sister or the sister in charge of the metabolic ward. The parents receive a simple explanation of the problem at the first consultation after the confirmation of the diagnosis and the start of treatment. At this time we concentrate on introducing the family and their health visitor to the unit and instructing them about the diet. When the parents return to the follow-up clinic the significance of the disorder and the nature of its inheritance are discussed. We have found that this information must be repeated two or three times in the first year, as it is seldom understood at the first consultation by the distressed and anxious family. At each outpatient visit the parents and the child see the dietitian, the paediatrician, and the liaison nursing sister.

\section{Ensuing good communications}

\section{LIAISON NURSING SISTER}

The post of liaison nursing sister was created some six years ago and is held by an experienced trained nurse who works from the hospital 
out into the community. She attends all metabolic clinics and gets to know the families who attend; she has authority to communicate with them, their doctors, and health visitors by telephone, and, when necessary, to visit by car, as she has a travel allowance for this purpose. This allows her to go with the local health visitor to the home or to visit the school when a child on a special diet is starting there. She has enough knowledge of the working of the unit to answer many questions herself, and if she cannot answer them herself she knows to whom she should refer the parent or doctor concerned. She also works closely with the dietitian and from time to time deals with some of the dietary problems.

At the outset we were concerned that such a liaison nursing sister might not be accepted by the health visitors in the region, but she has in fact been well received, and the staff in the region have appreciated her ability to help and instruct them in the nursing and supportive requirements of children with inborn errors of metabolism She has given several talks in the region to health visitors and midwives, who in their turn visit the hospital to meet her and discuss their individual problems. Telephone communication is regular but not too frequent; during the past two to three years the nursing sister and dietitian received about 500 calls a year, mainly for medical and social advice, and made a similar number of outgoing calls. The liaison nursing sister also makes about 50 visits a year to either homes or schools.

\section{GROUP ACTIVITIES}

The staff arrange parents' evenings and health-visitor discussions. For the past five years a party at Christmas has been held for all the children on special diets. There is a full variety of party fare on the table and everything can be eaten by the children. In this way it is possible to introduce the results of new recipes to the mothers. Each child is entitled to bring one sibling and both parents as guests, and as many as 150 parents and children have attended this Christmas party. Quite separately there have been more than 100 parents at our evening meetings. All these activities have helped to involve and interest the families of the affected children.

The unit paediatricians have spoken in every part of the region to doctors, health visitors, and midwives, explaining the reasons for the screening and the nature of the treatment and giving up-to-date reports on progress, and at the same time receiving lively observations and criticism. Precautions have been taken to ensure that the district community physician, the general practitioners, and health visitors are well informed about the progress of the individual children from a biochemical and intellectual viewpoint. In addition, the family know they may communicate with the hospital unit directly should they feel it necessary.

\section{RESULTS OF GOOD COMMUNICATION}

This concentration on all-round communication has had rewarding results.

Firstly the time between the first test and confirmation of the diagnosis is not more than six days. Secondly, the initial stay in hospital for children with PKU has been reduced from 16 to two days. This short stay allows us to confirm the diagnosis, start treatment, and introduce ourselves to the family. Thirdly, the need to bring the children back to the follow-up clinic has been greatly reduced. Children are seldom seen more than four times in each of the first two years, and possibly only twice or three times a year after that. Finally, only three children have been readmitted for restabilisation of diet during the past three years.

\section{Management}

Histidinaemia-A histidine-low diet ${ }^{4}$ (approximately $250 \mathrm{mg}$ histidine daily) is started in hospital during a 48-hour stay and maintained for the first two years of life to keep the plasma histidine concentration below $0.3 \mathrm{mmol} / \mathrm{l}$. The diet is stopped after the age of 2 years when speech is established and it is clear that mental and physical development are progressing well. The children attend the clinic every three months for clinical and biochemical assessment during the first two years and then at two yearly intervals for further review and for electroencephalography (EEG) and intelligence quotient (IQ) assessments.

Homocystinuria-When homocystinuria is diagnosed in the newborn period the infant is admitted to the hospital and given a trial with pyridoxine $200-300 \mathrm{mg} /$ day for seven to 10 days. If there is no biochemical response a low-methionine and high-cystine diet containing about $120 \mathrm{mg}$ of methionine daily is started and pyridoxine continued. This requires a three-week stay in the hospital. The patients are then reviewed every two to three months in the clinic and blood is taken for column analysis to measure plasma methionine and homocystine concentrations, as paper or thin-layer chromatographic methods are not sensitive enough for monitoring treatment.

Phenylketonuria-Most work has been with phenylketonuric children. Here biochemical supervision has been easier because the sensitivity of our method for plasma phenylalanine (Phe) estimation (thin-layer chromatography) allows measurement down to $0.1 \mathrm{mmol} / \mathrm{l}$, thus making it possible to use capillary blood taken by the health visitors and sent to us through the post. This blood is obtained weekly for the first six months of life, then every second week until the second birthday, and at monthly intervals thereafter for as long as possible. The policy with the phenylketonuric children has been to keep the plasma Phe concentrations below $0.4 \mathrm{mmol} / 1$ during the first two years and to allow a gradual increase up to $0.7 \mathrm{mmol} / \mathrm{l}$ over the next four to five years. Detailed amino-acid measurements by column analysis are made twice a year and at the same time serum calcium, phosphorus, alkaline phosphatase, proteins, and haemoglobin concentrations are determined. EEG examinations are made every second year, and IQ estimations at 2,4 , and 8 years.

\section{DIETARY MANAGEMENT OF CHILDREN WITH PKU}

The aim of the diet in children with PKU is to control the concentration of plasma Phe within the ranges mentioned earlier and at the same time provide for normal growth. Phe is provided from natural foods and the main bulk of the proteins from Phe-free preparations based on protein hydrolysates and amino-acid mixtures (Minafen, Cymogran, Albumaid XP, PK Aid 1, and Aminogran). Vitamin and mineral supplements are essential, and attention must be paid to the energy value of the diet and supplements of fat (Prosparol) and carbohydrate (Gastro-Caloreen) given when necessary.

In early infancy the required Phe is provided by liquid cows' milk. In the first instance $120 \mathrm{ml}$ (200 Phe) daily is given and the amount adjusted accordingly depending on the individual's tolerance for the amino-acid. Protein supplementation is with Minafen, Cymogran, or Albumaid XP. Older children are given their Phe from small amounts of milk, potato, other vegetables, and breakfast cereals and the protein supplemented with amino-acid mixtures (PK Aid or Aminogran). Low-protein bread biscuits and pasta and ample amounts of other carbohydrates and fats are given to ensure a satisfactory energy intake. High-protein foods such as meat, fish, eggs, poultry, cheese, and ordinary bread and biscuits are forbidden until the diet is appreciably relaxed.

\section{CLINICAL PROGRESS}

Detailed accounts of the progress of the histidinaemic and homocystinuric children will be published separately.

The general and emotional health of the children with PKU has been good and measurements of their heights, weights, and head circumferences have been within normal limits. Apart from a slight reduction in the $\gamma$-globulin component of the serum proteins and some slight reduction in the plasma tyrosine concentrations nothing abnormal has been seen. Intellectual development has been good; 45 children have reached their 4 th birthday, and of this number 18 are now beyond 8 years of age. Their intellectual development has been measured at 4 years and 8 years and the results assessed in relation to dietary control (table I). Although the numbers are small, it seems clear that continuous good control is paralleled by good intellectual

TABLE I-Comparison of IQ scores at 4 years and 8 years (18 children)

\begin{tabular}{|c|c|c|c|c|c|c|c|}
\hline \multirow{2}{*}{ IQ } & & & & \multicolumn{2}{|c|}{4 Years } & \multicolumn{2}{|c|}{8 Years } \\
\hline & & & & No & Average IQ & No & Average IQ \\
\hline $\begin{array}{l}81 \ldots \\
81-100 \\
101-120 \\
120\end{array}$ & $\begin{array}{l}\ldots \\
\ldots \\
\ldots\end{array}$ & $\begin{array}{l}\ldots \\
\cdots \\
\cdots\end{array}$ & $\begin{array}{l}\ldots \\
\cdots \\
\cdots\end{array}$ & $\begin{array}{l}3 \\
6 \\
6 \\
3\end{array}$ & $\begin{array}{r}78 \\
94 \\
110 \\
129\end{array}$ & $\begin{array}{l}3 \\
6 \\
4 \\
5\end{array}$ & $\begin{array}{r}80 \\
94 \\
111 \\
128\end{array}$ \\
\hline
\end{tabular}


progress and that the diet should certainly be maintained though with some relaxation beyond the 8 th birthday. In addition to the beneficial effect of diet we feel certain that a good family environment providing adequate motivation and stimulation has influenced the intellectual development of affected children in many cases.

\section{Laboratory arrangements}

We have deliberately emphasised the administrative arrangements for good communication with the outside world, but it is also important to emphasise the need for a consistently good laboratory service. There has been a close and careful integration of work between the screening and supporting sections of the laboratory. A few parttime laboratory scientific officers have chosen to confine their work to the screening laboratory but whenever possible the junior staff work in both areas, and timetables have been prepared accordingly. They have also been encouraged to work in a general routine laboratory for one day a week to obtain graded technician status. Some members of the staff have used day release and evening classes to work for higher qualifications and degrees. Two members of the unit have obtained PhDs, two MScs, and three technicians have obtained Higher National Certificates.

The turnover of staff has been small. Some have moved on to more senior posts and a few have left to go overseas or to return to routine laboratory work. We have not had the boredom or the staff wastage often attributed to screening units: on the contrary, we have maintained our supportive work and expanded our techniques for investigating individual problems such as organic acid, mucopolysaccharide, and lysosomal storage disorders and galactosaemia. The development of tissue culture of skin and amniotic fluid fibroblasts has allowed the unit to help in certain prenatal diagnostic problems and to continue its research activities into homocystinuria ${ }^{5}$ and methylmalonicaciduria. Recently a regional antenatal screening programme for PKU has been started, and now the unit screens upward of 35000 women a year.

\section{Costing}

LONG-STAY CARE

When an inquiry was made at long-stay mental hospitals to assess the cost of the care of untreated patients 54 patients with untreated PKU ( 24 men, 30 women) were found in an inpatient population of 3520 (an incidence of 1 in 65). Their average age was 39.5 years (range 25-47 in men, 19-64 in women) and their average length of stay in hospital 22 years (range 10-45). They were in good physical health. There had been four deaths in the previous five years in severely handicapped individuals, the cause of death being acute intestinal obstruction, pulmonary abscess, cerebral thrombosis, and acute peritonitis. It would, therefore, seem reasonable to allow 45 years as an average life expectancy of these patients.

\section{COSTS OF TREATING PKU}

There have been on average eight new cases of PKU a year since the screening of the whole region was started in 1971, and, using the same approach as in 1973,'2 a costing analysis for 1978 was worked out (tables II and III). The overall cost of screening in 1978 was $£ 19$ 275, which meant that each case of PKU diagnosed cost $£ 2409$. This time we assumed that two (not one) of the undetected children did not require special care because of low but not educationally subnormal intelligence or alternatively because of death in childhood or early adult life. This left six untreated children who would require special schooling and residential care in adolescence and adult life.

TABLE II-Cost of screening in 1978

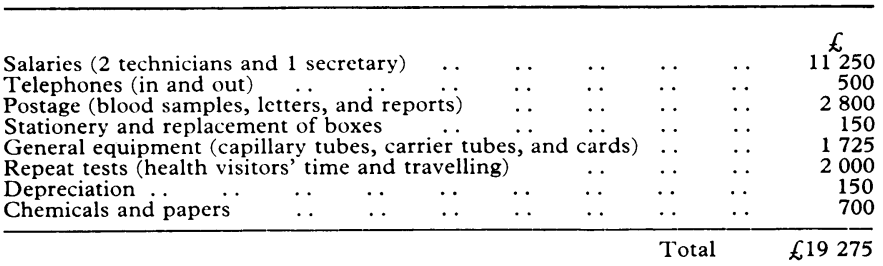

TABLE III-Cost of detecting each case and treating one patient for 10 years

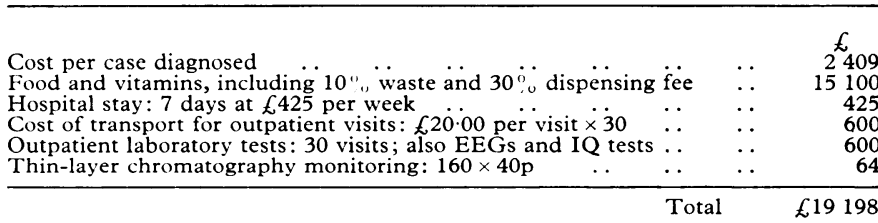

It has been possible to cost all the activities of this screening programme, including the salaries of the secretary and liaison nursing sister, the visits of the health visitor, and a proportion of the salaries of the consultant, dietitian, and educational psychologist relating to this work. There are figures for postage, telephones, laboratory running costs, inpatient and outpatient charges, and the special dietary products. The annual fee charges for normal schooling, special schooling, and residential adult care for the year 1978 have been obtained.

Tables IV and $\mathrm{V}$ show that the cost of long-term residential care far exceeds that of detection, treatment, and normal schooling, and we must add to this the benefit of alleviating the patient's suffering and that of his family and the positive contribution these individuals should be able to make to the economic and social life of the community.

TABLE IV-Cost of care of six untreated cases

\begin{tabular}{llr}
\hline $\begin{array}{l}\text { Average length of life (years) } \\
\text { Cost }(£):\end{array}$ & 45 years \\
$0-4$ years & & \\
$\begin{array}{l}5-14 \text { years } \\
6 \times 10(60) \text { patient years at } £ 1500 \text { a year } \\
15-45 \text { years } \\
6 \times 31(198) \text { patient years at } £ 3600 \text { a year }\end{array}$ & $\begin{array}{l}\text { home and special school } \\
\text { residential care }\end{array}$ & 90000 \\
\hline & & 712000 \\
\hline & Total & 802000
\end{tabular}

TABLE $\mathrm{V}-$ Balance sheet of costs of detecting and treating eight cases of $P K U$ versus costs of untreated PKU

\begin{tabular}{|c|c|c|}
\hline \multirow{6}{*}{ 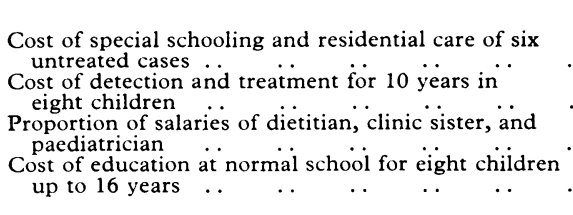 } & $£$ & $£$ \\
\hline & & 802000 \\
\hline & 154000 & \\
\hline & 3000 & \\
\hline & & \\
\hline & 76000 & 233000 \\
\hline & lance & 569000 \\
\hline
\end{tabular}

\section{The future}

The emphasis in this paper has been on caring and on the awareness that the families of children with metabolic disorders require continuing support while the children are growing up. The need for support, which will continue into late adolescence and early adult life, is particularly important for the girls. The problems of marriage and pregnancy, with the need for further genetic counselling and advice about the significance of maternal $\mathrm{PKU}^{6}$ and appropriate clinical supervision, will require the additional help of obstetricians and physicians.

Ideally the regional team should include a physician with an interest in genetics, as well as the paediatrician, and the two would share the responsibility for the care of these patients and families. In this way both physicians would help the patients during the emotionally stormy period of adolescence, while the paediatrician would have the opportunity to maintain continuity with his patients in adult life in order to learn the outcome of the early treatment and the care that he has offered them as children over the years. The physician would in his turn learn about the handling of their problems in childhood and meet the patients for whom he will become responsible.

Our determined attempts to improve communication and to 
go out into the community to describe and explain the unit's work has given the authorities and the families confidence in the unit and a realisation that it is concerned with them as people and not just with the results of the screening tests and the treatments prescribed. We believe that this, to a considerable extent, has been achieved by the creation of a liaison nursing sister post and with the invaluable wholehearted help of those regional health visitors who have been so keenly interested in the programme.

We are indebted to the health visitors in the North-western Region for their help and enthusiasm, to Mrs M A Riley for the psychological assessments, and to Miss Pat Chapman and the unit staff, who spent much time in acquiring the information on which the costing analysis was based.

\section{References}

1 Sardharwalla, I B, et al, Annals of Clinical Biochemistry, 1972, 9, 126.

2 Komrower, G M, Pediatrics, 1974, 53, 182.

3 Scriver, C R, Davies, E, and Cullen, A M, Lancet, 1964, 2, 230.

${ }^{4}$ Dryburgh, E H, Sardharwalla, I B, and Komrower, G M, Archives of Disease in Childhood, 1976, 51, 236.

${ }^{5}$ Fowler, B, et al, fournal of Clinical Investigation, 1978, 61, 645.

${ }^{6}$ Komrower, G M, et al, British Medical fournal, 1979, 1, 1383.

(Accepted 26 fuly 1979)

\title{
Patients' reactions to their investigations: a study of 504 patients
}

\author{
CLIFFORD HAWKINS
}

British Medical fournal, 1979, 2, 638-640

\section{Summary and conclusions}

Five hundred and four patients who had undergone hospital investigations were interviewed to find out how much information they had been given about the tests; their reactions before, during and after the test; and any after effects. In $74 \%$ of cases the tests had been satisfactorily explained. Patients were told more about complicated procedures such as cardiac catherisation than about routine ones such as venepuncture or barium meal examinations. The comments doctors made while performing the investigations were generally reassuring and were only rarely worrying or impatient. About half the patients suffered pain or discomfort during the test and rather more complained of after effects. Only $5 \%$ of patients said they would refuse the test again, though $36 \%$ said they would agree only reluctantly.

Communication lies at the heart of the problem. Hospitals should consider issuing handouts on investigations to back up the doctor's information and to dispel myths. Staff should be more careful in concealing frightening-looking equipment, and if patients have to wait during investigations it may help reduce their anxiety if they are provided with something to occupy their time.

\section{Introduction}

In many specialties advances in diagnosis have preceded those in treatment. Investigations are often uncomfortable, sometimes painful, and occasionally dangerous, ${ }^{1}$ apart from their great and increasing cost to the NHS. Yet little is known about their effect on patients. Although much has been written about communication with patients, ${ }^{2-5}$ the lack of information on patients' reactions to investigations is surprising, especially as failure to inform patients may have medicolegal consequences.

Queen Elizabeth Hospital, Birmingham B15 2TH CLIFFORD HAWKINS, MD, FRCP, consultant physician
This study was planned to find out what patients knew about their investigations, how much anxiety they had before and during them, and to what extent they suffered physical discomfort.

\section{Patients and methods}

Inpatients who had undergone investigation were interviewed using a coded questionnaire with 65 questions. Their mental clarity was also recorded.

Interviews were carried out over three years by three interviewers; none had special training in the technique of interviewing, though each had spent her professional career in this field, one being head of a personnel department and the other two working in psychiatry. Each dressed informally and made it clear to the patient that her object was to obtain a detached and independent opinion and that she was not part of the establishment. Interviews took place in the hospital, generally in the ward, but where there was no possibility of others overhearing.

The questionnaire was divided into three parts, covering before the test, during it, and afterwards. Patients were asked: before the interview -whether the test was explained and by whom, whether the patient understood and knew where it was going to be done, and whether he was frightened or terrified (using a mood adjective check list); during the test-how much of the procedure was remembered, whether any discomfort, pain, or other effects were noticed, and whether any comments were heard-either reassuring, worrying or irrelevant; after the test-whether the patient would have the test repeated (no, yes, or reluctantly); whether the test affected the patient in any way (better, no change or worse), and whether any suggestions could be offered for making the test easier for other patients.

The answers were put on to IBM cards and analysed by a punch card sorter.

\section{Results}

Five hundred and four patients from 13 medical and eight surgical wards were interviewed; 462 were chosen by random selection and 42 otherwise included. They were almost equally divided between the sexes: 264 men and 240 women. Their age range is given in table I. Four hundred and seventy-six were mentally clear, and 28 were slightly confused or had a speech difficulty though not sufficient to prevent them being satisfactory witnesses. Four hundred and fifty-seven were United Kingdom subjects, seven were from Eire, four from Europe, and 36 were immigrants; a language problem occurred in 14 but measures were taken to ensure that questions were understood. The 